\title{
LAS CONDICIONES DE ÉXITO EN LA CONSTRUCCIÓN DE LAS
} RUTAS ALIMENTARIAS

Ernesto Barrera $^{1}$

Universidad de Buenos Aires

barrera@agro.uba.ar

Material original autorizado para su primera publicación en la revista académica REDMARKA. Revista Digital de Marketing Aplicado.

https://doi.org/10.17979/redma.2013.01.010.4756

Recibido: 22 Abril 2013

Aceptado 13 Junio 2013

\section{Resumen}

El término patrimonio es idóneo para identificar el territorio y por extensión a ciertos alimentos, sugiere una proyección temporal larga y plantea la imagen de un recurso complejo que tiene sólidas bases en el pasado y se proyecta hacia el futuro.

Se propone que las rutas alimentarias, con base en el patrimonio alimentario, conformen una oferta turística que aporte a su posicionamiento, contribuya a impulsar su imagen y demanda, en un negocio inclusivo que impacte en las comunidades. El patrimonio se configura históricamente en la interacción de las clases y sectores sociales del cluster productivo dando origen al paisaje cultural de la ruta. Un producto auténticamente representativo no debería excluir a parte de sus protagonistas.

${ }^{1}$ Coordinador del Área de Turismo Rural de la Facultad de Agronomía de la Universidad de Buenos Aires. barrera@agro.uba.ar 
En este artículo se revisa la bibliografía sobre las rutas alimentarias para determinar los principales factores que condicionan el éxito de las mismas, poniendo énfasis en que deben reflejar el carácter inclusivo que requiere la situación social de América Latina, sin que ello suponga que en otras regiones deba ser diferente.

Los resultados proponen analizar nueve factores para la determinar el carácter exitoso de una ruta alimentaria: Coordinación de la ruta, apoyo del gobierno, la masa crítica de la oferta, la diversidad de las experiencias ofrecidas, su calidad, la consistencia con el tema de la ruta, la oferta de suvenires, la demanda del mercado y su carácter inclusivo.

Un punto central en el análisis, es la preocupación sobre quién se beneficia con el desarrollo de las rutas alimentarias. Las rutas con pretensiones de exclusividad, frecuentemente lo son a partir de cierto carácter glamoroso que excluye a los actores más indefensos del paisaje cultural de la ruta.

Se alerta en las conclusiones que las configuraciones no inclusivas de las rutas alimentarias llevan implícito un elevado riesgo, más aún en los países de América Latina signados por los conflictos agrarios.

Palabras clave: patrimonio, ruta alimentaria, negocios inclusivos

\section{Abstract}

CONDITIONS FOR SUCCESS IN THE CONSTRUCTION OF FOOD TRAILS

The term heritage identifies the land and, thus, certain food. It refers to a complex long term resource that is strongly grounded in the past but that it is also forecasted into the future.

The goal of this paper is to enhance food heritage and shape food trails into a community inclusive business through tourism positioning and the development of their image and demand. Heritage is the result of the interaction between the productive clusters' different social groups, and provides the cultural resources for each food trail. A truly representative product is inconceivable without its main social actors. 
This article conducts a literature review with the aim of identifying the key drivers of food trails success, and emphasizing their inclusive approach which is particularly, but not only, required in Latin America. Nine main factors were identified: coordination of the route, government support, critical mass of supply, diversity of experiences offered, quality, topic consistency among a route, offering souvenirs, market demand and inclusiveness.

A main issue on this analysis is the definition of who should be benefit by food trails' development. Trail intended to be exclusive, usually disregard the most vulnerable actors from their cultural heritage. Moreover, not inclusive trail may be harmful to the community, and even more in Latinamerica, a region signed by agrarian conflicts.

Keywords: heritage, food trail, business inclusive. 


\section{LA DINÁMICA DE LA IDENTIDAD PLURAL EN LAS RUTAS ALIMENTARIAS}

"Dime qué comes y yo te diré quién eres" este famoso aforismo de BrillatSavarin (2005) refiere a los factores de identidad de la alimentación en relación con categorías sociales de los parisinos.

Lo que comemos no se corresponde en forma simplista con los ingresos de los consumidores. Los obreros franceses comen pâté de campagne - un plato barato, dilecto entre los franceses de clase trabajadora -no porque sea el mejor pâté que se pueden dar el lujo de pagar, sino porque lo que nos gusta comer está determinado por nuestra educación gastronómica.

"Los obreros prefieren el gusto del pâté de campagne al de otros pâtés o platos más costosos, tales como la terrine de foie gras, porque un obrero es socializado en los gustos de la clase obrera. Cuando un obrero francés come pâté de campagne, está afirmando con ello (tanto para sí como para los otros) que él es un obrero". (Tobin J., 2002).

Aunque debe señalarse quela práctica y la voluntad modifican el gusto, es decir éste no sería sólo producto e indicador de clase social (Bourdieu, 1998).

"El bife con papas fritas es antes que nada francés, es el plato que identifica a un obrero y a un patrón" es un plato más nacionalizado que socializado. (Barthes 1972: 63).

En la definición del gusto, existe cierta preeminencia de los valores definidos por el territorio por sobre los que definen las clases sociales. El gusto es uno de los recovecos en los que la identidad da batalla a la globalización y lo hace en un sentido socialmente inclusivo indicando que la categoría de nación aún está viva.

Los factores de identidad que anidan en un alimento, si bien no dejan de reflejar cierta pertenencia de clase, expresan con prelación los de la nacionalidad. Un alimento que refiere de inmediato a la nacionalidad argentina 
es la yerba mate; se consume en todo el país y lo hacen todas las clases sociales.

Un territorio, al interior de una nación, también tiene su identidad propia, la identidad local. Algunos alimentos tienen la identidad de un territorio más reducido, por caso, la mandioca y el maíz en la Argentina, son muy consumidos en las provincias del norte y escasamente utilizados en la gastronomía de la Patagonia.

El término patrimonio es idóneo para identificar el territorio pues sugiere una proyección temporal larga. Plantea además la imagen de un recurso complejo, cuyos componentes forman un sistema: el ecosistema artificializado, las infraestructuras, las interacciones sociales y los conocimientos, las representaciones y los valores que le dan estructura. En este sentido el patrimonio es memoria colectiva, a la vez registro de acontecimientos y experiencias pasados y sobre todo, capacidad de proyección hacia el futuro. (Linck, 2006).

El mismo autor sostiene que la patrimonialización de los recursos territoriales se relaciona con la construcción de dispositivos de apropiación colectiva, destinados a fijar los derechos de uso y de manejo y a establecer la responsabilidad de sus depositarios hacia los territorios y la sociedad. El caso más concreto de apropiación del territorio en un alimento es el de las Denominaciones de Origen.

Las identidades, de alguna manera una extensión del patrimonio territorial, no son mundos cerrados ni se presentan uniformadas, ni permanecen estáticas. La identidad no se cristaliza, la identidad no es la tradición, es por el contrario dinámica. Como afirma Carlos Fuentes, "la identidad es lo que somos ahora mismo"

El patrimonio tiene un carácter dinámico, muy alejado de los planteamientos que tienden a relacionarlo con la tradición resaltando su carácter estático, pretendiendo lastrar en el pasado el lógico devenir al que están sometidas todas las sociedades y todas sus producciones culturales (Aguilar Criado, 2005). 
Abordar con los mismos instrumentos que satisfacían a los consumidores de la primera mitad del siglo $\mathrm{XX}$ a los consumidores del siglo $\mathrm{XXI}$ promete dificultades. Los cambios que se han producido en las preferencias de los consumidores son estructurales. Muchos alimentos que eran buenos entonces ahora parecen tóxicos, tienen elevado tenor graso, excesos de sal, etc.

Probablemente las dificultades que enfrentan las Denominaciones de Origen [DOC] en el mercado mundial, especialmente las de vinos, tengan origen en que han pretendido inmovilizar en un protocolo de calidad lo que por naturaleza es dinámico.

América presenta hoy un tejido intercultural extremadamente complejo y mestizo. Este tejido se nutrió de las poblaciones indígenas originarias, de las poblaciones inmigrantes que se establecieron en la época de la Conquista y la Colonia, y se alimentó con sucesivas olas de inmigración de millones de personas de origen europeo, africano y asiático, en las que destacan las olas en la segunda mitad del siglo XIX y la primera mitad del siglo XX. Ello atraviesa toda la cultura latinoamericana $y$, como señalaba Arciniegas a ciertas élites, "Por más que nos creamos españoles o franceses, somos americanos, el continente nos imprime un aire, un acento, una luz, un color..." (Castedo, 1999). Y, corresponde aquí decirlo, una comida.

El alimento sigue los senderos marcados por la identidad. Una historia de conflictos y sinergias entre comunidades indígenas, conquistadores, corrientes de inmigrantes y ahora también de emigrados, los que en cada regreso traen lo suyo.

Durante la Conquista, los españoles prohibieron algunos alimentos nativos como la quinoa por ejemplo, introdujeron forzosamente otros, como el trigo y el mestizaje dio origen a otros, como el chocolate o el tequila.

Existen alimentos y comidas que tiñen al conjunto de un país, pero estas señas identitarias no inhiben las expresiones diferenciadoras. En la Argentina el asado y la empanada son totalizadores, pero las diferencias territoriales son importantes; en Ecuador el maíz define la identidad nacional, pero el cuy -un pequeño roedor herbívoro que las amas de casa serranas crían en las cocinas 
con los desechos de vegetales y comida -, emblemático de las sierras, y aborrecido en la costa no es menos ecuatoriano.

La historia de la alimentación es tributaria de la dotación de recursos del territorio -vegetales, animales y minerales, suelo y clima, de las tecnologías disponibles en cada momento [irrigación, labranza, mejoramiento de variedades en fauna y flora, introducción y aclimatación de especies importadas, modos de conservación y acondicionamiento de los comestibles] y de la historia económica y social del territorio. Cada territorio tiene una singular combinación de estos factores que lo diferencia y singulariza patrimonialmente. (De Certeau, 2000)

El territorio ha adquirido valor de mercado de la mano de la globalización al revelar la convivencia de las tendencias a la homogeneización con la heterogeneización como partes indisolubles de la misma realidad global (Aguilar Criado2005) o, en otros términos, como la coexistencia de la tipicidad con la generización.

El mercado de los alimentos ofrece es un interesante campo de análisis: las preferencias de los consumidores cambian permanentemente obligando a los productores a modificar procesos productivos y formulaciones de producto, las DOC constituyen una barrera que quita reflejos de adaptación, un problema europeo (en rigor mediterráneo) como se dijo especialmente visible en el mercado de vinos.

Por otro lado, la creatividad de los jugadores dominantes del mercado también juega su juego. Cuando parece que todo está dicho, quienes han promovido la innovación en una dirección, señalan otra nueva según nuevos descubrimientos, muy convenientes al statu quo.

Décadas de promoción de la producción orgánica promovidas por los países desarrollados y los organismos internacionales como una salida para los productores agropecuarios, con la promesa que encontrarían una demanda ansiosa en los consumidores europeos preocupados por la preservación de la naturaleza a escala planetaria, estarían llegando a su fin con la aparición de un nuevo movimiento que promueve los Alimentos en el $\mathrm{Km}$. $\mathrm{O}$ o Food Miles. 
El nuevo concepto parte de la premisa que si los alimentos reducen la cantidad de Km. que deben viajar desde la finca del productor a la mesa del consumidor se reduce el consumo de energía y de las emisiones de dióxido de carbono en la atmósfera, estaríamos por lo tanto frente a un modelo globalmente más sustentables que el de la producción orgánica. Un modelo que favorece a los productores de los países más desarrollados.

Los fundamentos del nuevo movimiento ambiental Food Miles ofrecen varias inconsistencias y no sería posible afirmar que un alimento comprado por un consumidor europeo a un productor local sea menos dañino para la naturaleza y la capa de ozono que otro comprado un productor americano.

Aunque no nos extenderemos en el tema, algunos de los puntos flacos surgen del alto nivel de emisiones de gases de efecto invernadero que provocan los subsidios del primer mundo a su agricultura, los desequilibrios comerciales que impiden la realización de los beneficios de la liberalización del comercio de biocombustibles y el impacto negativo sobre la reducción de la pobreza del tercer mundo. (Desrochers y Shimizu, 2008)

Aún con las prevenciones realizadas sobre los diversos distintivos de calidad promovidos por los países del primer mundo, cabe señalar que las DOC y otro tipo de referencias de calidad pueden jugar un rol valioso para varias producciones de América Latina ayudándolas a competir en el mercado mundial.

La naturaleza, el territorio y sus tradiciones dan significado simbólico y favorecen la inserción en nichos de mercado de los alimentos que por su carácter histórico, o por exitosas estrategias comerciales, consiguen embeberse de esa identidad. Las rutas alimentarias juegan un papel importante en ambos casos, validando una imagen o construyéndola.

El vínculo entre las rutas alimentarias y las indicaciones geográficas no es caprichoso. Vinos y Quesos son los alimentos que acumulan más distintivos de calidad referenciados territorialmente y son también ellos los que muestran mayor cantidad de rutas alimentarias desarrolladas. Algo similar puede decirse 
de los distintivos de orden religioso o étnico. Todos ellos pueden enriquecer las rutas alimentarias.

En esencia, las rutas alimentarias como reflejo del territorio, deben ofrecer el patrimonio vivo y actual e ilustrar el camino recorrido hasta el presente. Son por su naturaleza territorial, un producto dinámico y plural pues el territorio, tanto cuando se expresa comarcalmente, o cuando lo hace como expresión nacional, es totalizador, expresa todas las clases y sectores de la sociedad. Es por definición una categoría inclusiva.

\section{ASPECTOS ECONÓMICOS DE LAS RUTAS ALIMENTARIAS}

Existe una amplia literatura internacional sobre las rutas alimentarias en las que se consideran diversos económicos, sociales y ambientales. Barrera y Staniak (2012), Briedehhann (2004), Elías 2006, Frochot (2000), Hall (2004, 2012), Hall y Mitchell (2000), López-Guzmán Guzmán (2008), Mendelson y Quick, D. (2009), Renting et al, (2003), Schlüter (2002).

La Organización Mundial del Turismo ha considerado especialmente el tema de los alimentos locales y su vínculo con el turismo y en el 2000 dedicó la Conferencia Internacional de Chipre al tratamiento del tema en el que destacan los trabajos sobre el tema de Mr. Thord Ohlsson y de Dr. Isabelle Frochot (WTO-CTO, 2000)

La revista Pasos (2008) ha dedicado un número exclusivamente a la temática del turismo gastronómico y del enoturismo.

La temática concita un creciente interés en América Latina, desde 2005 en la Facultad de Agronomía de la Universidad de Buenos Aires se realiza anualmente un curso internacional sobre Rutas Alimentarias y Turismo del Vino.

Aunque el turismo se percibe como una de las pocas opciones viables para el desarrollo en los países afectados por pobreza rural, varios autores y ya desde hace tiempo vienen advirtiendo que obligados por las presiones de la reestructuración, e impulsados por la demanda de creación de empleos, los gobiernos de los países en desarrollo con frecuencia son víctimas de los 
peligros del desarrollo al azar, sin tomar en cuenta la situación económica y cultural de las comunidades rurales, la conservación medio ambiente o la inclusión de los residentes locales en la toma de decisiones (Haywood, 1988; Long, 1990; Prentice, 1993).

En el mismo sentido, los países en desarrollo con frecuencia son víctimas de los peligros de la políticas de desarrollo ausentes o improvisadas, sin tomar en cuenta el desarrollo económico y el bienestar cultural de las comunidades rurales, la conservación del medio ambiente o la inclusión de los residentes locales en la toma de decisiones (Briedenhann y Wickens, 2004) .

Desde la década de 1990, la evolución de las tendencias del turismo ha cambiado, apartándose del turismo de masas estandarizado hacia patrones más personalizados y flexibles. En el marco de estas tendencias, el Turismo Rural tiene una gran posibilidad de desarrollo. Se argumenta que los turistas rurales, en particular, tienen una amplia gama de motivaciones, de índole ecológica, de aventura, de interés cultural o de la paz y la tranquilidad del campo (César Dachary y Arnaiz Burne, 2006).

El impacto económico de las rutas turísticas en Europa ha sido significativo. Una de las rutas más conocidas es religiosa, el Camino de Santiago, que está generando una importante derrama en Galicia. En este artículo abordaremos las rutas nacidas en torno a los alimentos.

El alimento nace de la tierra, fruto del trabajo de los agricultores; es junto a las fibras el principal producto de la ruralidad. Cuando alimento y turismo se reúnen, éste lo valoriza y con ello, acrecienta el valor del trabajo de los actores de la cadena gastronómica, aunque en general premia sólo marginalmente

\section{a los agricultores.}

El uso del vocablo "alimentarias", que es incorrecto en lengua castellana lo utilizamos para diferenciar la propuesta de las rutas gastronómicas, entendiendo que la palabra gastronomía referencia -al menos en los usos y costumbres- más al restaurante y a la cocina que al agricultor, como lo evidencian numerosas rutas gastronómicas que no son más que una oferta reducida a la comida. 
El alimento se relaciona fuertemente con el turismo pues éste requiere de aquel para viabilizar la experiencia turística. Es, la más de las veces, un facilitador, un acompañante necesario de la misma, pero cuando alcanza notoria identidad puede ser un protagonista del turismo.

Las rutas alimentarias modifican el carácter facilitador de los alimentos, brindándoles espacio para el lucimiento y posicionándolos en el mercado. Elevan así su demanda y su precio; van más allá del recinto gastronómico mejorando también la economía de los productores agropecuarios. Por eso las visualizamos como una vía para aumentar la biodiversidad, la oferta manducatoria de los pueblos e incorporar nuevos productos al mercado.

\section{La creación de una ruta requiere que se desarrolle una oferta turística creativa que presente el pasado y que ofrezca la posibilidad de obtener nuevas experiencias sin desnaturalizar el soporte cualitativo del alimento.}

Durante la última mitad del siglo $X X$ se ha producido un cambio estructural en el modo de apreciar la comida por parte de los turistas. Si antes los viajeros no estaban interesados en los alimentos extraños de las regiones que visitaban, ahora buscan nuevas experiencias y prueban los platos con colores y sabores locales, convirtiendo así al turismo en un fantástico campo de experimentación apto para la introducción de nuevos productos en el mercado.

Las industrias culturales -de las cuáles el turismo y, crecientemente, los alimentos- son ejemplos, evidencian un fuerte crecimiento; es así que los recursos locales e indígenas adquieren un nuevo y revalorizado posicionamiento y por tanto un rol más importante en el proceso de desarrollo de las regiones atrasadas.

El aislamiento de las regiones rurales y de las economías tradicionales tuvo un doble efecto: postergó a sus habitantes, pero también conservó las tradiciones, manteniéndolas, muy probablemente, a contramano de las aspiraciones de progreso que la población asocia a la apertura de la economía y de la cultura. Esas tradiciones, puestas en los alimentos, revelan un panorama complejo compuesto de ruralidad, rusticidad, calidad, pureza, autenticidad; conceptos que en el imaginario de los consumidores citadinos se asocian positivamente 
porque los aproxima al pasado que valoran, al patrimonio perdido. ¡Lo indeseable muto en patrimonio!

La herencia patrimonial que el progreso y la apertura de las economías dilapidaron adquiere ahora, por el principio de la escasez, una nueva dimensión, un valor del que carecía. Y aunque para los residentes locales esa tradición algunas veces sólo evidencia su retraso relativo, para los citadinos la herencia conservada puede ser atractivamente glamorosa. (Mc Crone, 1995.)

El alimento y la gastronomía local tienen un lugar al lado de los museos, monumentos y otros recursos patrimoniales; el turismo ofrece al alimento una vía para diferenciarse, en tanto éste le ofrece al turismo la oportunidad de ampliar su oferta y romper la estacionalidad. Asociados, alimentos y turismo, pueden recorrer un camino más prometedor para las regiones postergadas del mundo rural.

El diseño las rutas alimentarias requiere que se considere la naturaleza y el carácter económico del vínculo entre alimento y turismo.

El turista se asoma a los territorios que visita con una perspectiva más compleja que la de su propio mundo individual, generando una relación con el destino (y con sus objetos y mercancías) que independiza su valor de los costos de producción. (MacCannell, 2003)

La perspectiva de MacCannell se vincula a la de Marx que define a las mercancías como fetiches que adquieren en el mercado vida propia con un precio que se determinan con independencia de su valor.

El turismo amplía la brecha entre valor y precio re-significando la mercancía. Las rutas alimentarias juegan ese rol sobre los alimentos, los fetichizan separando su precio del costo de producción.

Esta separación a veces es muy significativa. Con frecuencia el turista paga más por un alimento comprado directamente a su productor que en el supermercado de la ciudad donde reside.

El consumo de los alimentos es siempre un hecho cultural y cuando se produce durante las vacaciones la nostalgia puede perdurar para siempre. El turista no 
consume una dieta de proteínas, grasas e hidratos de carbono, sino que devora, con el alimento, otra que contiene paisajes, historias, anécdotas y el saber hacer del pueblo que visita. ¿Quién puede definir el valor de estos imaginarios?

\subsection{Beneficiarios directos de las rutas alimentarias}

El desarrollo de las rutas alimentarias puede ser visto como una oferta sustentada en los emprendedores y empresarios locales que encuentran en el turismo una forma de mejorar sus ingresos sobre la base de sus recursos básicos o más característicos de la región. (Mason 2010)

Las rutas se inician sobre la base de productos que expresan la síntesis de la historia alimentaria y productiva de un territorio, las que según la visión de Luce Giard (De Certeau, 2000) integran los recursos naturales del territorio, las tecnologías y su historia económica y social, mirada que se corresponde con la de los SIAL (Muchnik, 2010)

El desarrollo de las rutas alimentarias es relativamente reciente, han surgido en las dos últimas décadas del siglo $\mathrm{XX}$, en Europa y constituyen una forma de construir redes que aproximen a los productores a los consumidores (Hall, 2012).

Las rutas son promovidas por los gobiernos y generan el interés del sector privado por varias razonas entre las que se destacan:

- La gastronomía orientada al turismo tiene una alta rentabilidad,

- El turismo de los alimentos y el vino puede relacionarse fácilmente con otros productos turísticos culturales y naturales, lo que permite que el destino tenga una oferta más amplia y comprensiva,

- Las zonas rurales afectadas por su retraso económico cuentan con una alternativa: la reactivación, el desarrollo o el mantenimiento de los alimentos con identidad local, y

- Los barrios suburbanos de origen rural puede llegar a ser atractivo para los visitantes, especialmente aquellos que se especializan en 
determinados alimentos étnicos. Una concentración o restaurantes, cafés y mercados puede aportar carácter a los mismos (Richards, 2012).

La relación entre la alimentación y el turismo en términos productivos debe integrarse en una estrategia de desarrollo económico local que trate de maximizar la relación económica y social entre los productores y la industria del turismo.

Pero veamos qué es una ruta alimentaria. Son itinerarios turísticos basados en alimentos con identidad territorial en torno a los cuáles se construye una oferta recreativa, lúdica y cultural que permite al visitante explorar la cadena de valor del alimento, desde la producción primaria hasta el plato. Ofrecen una posibilidad de desarrollo inclusivo y contribuyen al posicionamiento y la promoción de los alimentos identitarios. Reflejan en su organización el patrón complejo del territorio rural que les da origen y cuidan la calidad tanto desde la perspectiva de la salubridad como de la identidad territorial. (Barrera y Staniak, 2013)

La propuesta incorpora a productores primarios, restaurantes, agroindustrias, hoteles y otros agentes económicos y sociales que varían según las características del alimento o la región.

Analizar la perspectiva del turista requiere precisar la figura del turista de los alimentos, algo que obliga a diferenciar entre aquel que consume el alimento de la ruta como parte de la experiencia del viaje y otro que ha realizado la elección del destino motivado por la ruta.

El turista que viajó motivado por la ruta y que busca realizar un recorrido histórico por el territorio, visita a productores, industrias, participa en festivales de alimentos, concurre a restaurantes y sitios específicos para realizar una degustación o una experiencia sensorial con sus alimentos. (Hall y Mitchel, 2011, citado en Hall 2012).

Sin embargo los turistas que recorren una ruta alimentaria tienen un perfil más amplio. Además de los turistas que viajaron motivados por la ruta, otros aprovechan las propuestas que ésta ofrece a todos los que visitan el destino con independencia de sus motivaciones, pues "chocarán" con la ruta en los 
restaurantes u otros sitios. Desde una perspectiva económica y social interesan el conjunto de turistas que se relacionarán con la experiencia de la ruta.

Todos los turistas que enfrenten una oferta organizada recibirán su impacto, hayan tenido o no como motivación primaria para el viaje, visitar la ruta.

Que las industrias se beneficien de las rutas alimentarias depende de una serie de factores económicos, sociales y políticos entre los que destacan el grado de articulación intersectorial de la economía, el patrón de gasto de los visitantes y su derrama.

La cuestión de quién se beneficia debe ser fundamental para la evaluación de las políticas regionales de desarrollo y estrategias económicas cuando se considera el papel de las rutas alimentarias. (Hall ,2012)

Son numerosas las rutas que por carecer de una buena planificación y una eficiente gestión son rutas muertas. (Frochot, 2000), tanto para las pequeñas como para las grandes empresas de alimentos el turismo puede ser muy importante en términos de desarrollo de marca pero el éxito de los emprendimientos particulares requiere de la planificación territorial.

En la planificación territorial, el turismo debe considerarse como un aspecto más del desarrollo global del negocio de la producción de alimentos más que como una estrategia en sí misma.

Uno de los principales beneficios que reportan los pequeños productores agropecuarios es el ingreso turístico que le genera la ruta, que opera como un destino, y la vía que ofrece la para escaparle a las cadenas de comercialización.

Para el sector industrial las principales ventajas dependen del tamaño de la empresa, para las más pequeñas el principal beneficio puede estar dado por la facturación directa, no paga costos de distribución y además es al contado. Para las grandes empresas el mayor beneficio pasa por la fidelización de sus clientes. 
En orden a considerar los beneficios que puede acarrearle una ruta alimentaria al sector turístico tradicional y al comercio en general, cabe apuntar los siguientes:

- El alto perfil de exposición que pueden lograr la gastronomía y algunos alimentos como atractivos turísticos puede proveer oportunidades para otros sectores turísticos.

- La imagen de la región gana asociada a productos locales y a la gastronomía de calidad

- La ruta alimentaria es una atracción en sí misma que amplia el rango de motivaciones para visitar el destino.

Para Frochot uno de los valores importantes de la ruta es la sinergia que se genera entre los diversos operadores que la integran. Operadores ampliamente distribuidos a los que lograr la visibilidad que les brinda la ruta de manera individual les resultaría muy oneroso.

\section{LAS CLAVES DEL ÉXITO}

El papel del Gobierno parece ser un aspecto muy importante, al menos como detonante, tema sobre el que varios autores coniciden (Hall, 2012; Frochot, 2000; Mason, 2010).

Otro factor de éxito en el turismo es la singularidad de la oferta. Por eso recomendable que los destinos no copien lo que han hecho otras regiones, sino que cada uno se enfoque en resaltar e incrementar las características que le brindan esa distinción.

Otro factor es el desarrollo de atractivos que aseguren que los visitantes lleguen, gasten y permanezcan buen tiempo en el destino.

En el corto plazo el enfoque central en la construcción de una ruta debe ubicarse en cómo las empresas cooperan entre sí y cómo el gasto circula en la economía local. Esta estrategia inicial enfatiza las compras locales y el fortalecimiento de las marcas locales (Hall, 2012) a lo que debería sumarse un gran trabajo de coordinación en la construcción participativa del proyecto. 
En el mediano plazo el foco ha de ponerse en el desarrollo de las redes y en el fortalecimiento de las relaciones entre las empresas y actores locales porque estas redes influirán en el movimiento de los visitantes y en el gasto que realicen. En esta etapa el énfasis debe ponerse en que los actores comprendan y adopten el proyecto como propio. Este proceso requiere que se realicen numerosas reuniones y talleres de trabajo participativos, un objetivo de ellos es detectar a los individuo líderes y a las empresas que "marcan el camino".

En el largo plazo el foco debe ponerse en el desarrollo de capital intelectual. La creación de redes -relacionadas al capital económico y social- asegura una base económica diversa para que la ruta no dependa exclusivamente del turismo. El capital intelectual cobra valor porque apoya el desarrollo de las marcas, aspecto que puede brindar la principal motivación de participación para algunas empresas.

\section{Los productos o mercados turístico específicos deberían ser} considerados como un medio y no como un fin en sí mismo. Las rutas alimentarias deben ser integradas a una estrategia de desarrollo regional, más que planteadas como una estrategia aislada. El capital social e intelectual puede ser tan y aún más importante que la infraestructura física.

Para ser exitoso se debe ser realista respecto del aporte al desarrollo económico regional de las rutas alimentarias, esto implica ser consciente de los problemas de estacionalidad así como de evaluar objetivamente la cantidad de gente que puede ser atraida a la región.

La colaboración entre las empresas y el desarrollo de redes desde el productor hasta el restaurantes y los consumidores son otro aspecto crítico.

La finalidad comercial de las rutas alimentarias debe centrarse en que la gente permanezca en la ruta el mayor tiempo posible, gaste localmente y que siga haciéndolo, comprando sus productos, una vez que regresó a su hogar, objetivo que lleva la ruta desde su región hasta el lugar de residencia de los turistas. Hasta allí se debe llegar con los alimentos, por eso el suvenir tiene una importancia significativa en el desarrollo de las rutas alimentarias. 
El principal producto del desarrollo de las rutas alimentarias es la creación de capital social y éste no puede ser apropiado en exclusividad por las empresas, va de suyo que el desarrollo implusado por el sector privado es, cuanto menos, muy difícil. Un camino en esta dirección sería el desarrollo de esfuerzos cooperativos, sin embargo es difícil excluir de los beneficios a quienes no aportan. El rol del Estado como impulsor de las rutas es, por tanto, decisivo.

Mason (2010), Hall (2012) plantean algunos aspectos que hace al éxito de una ruta alimentaria. Barrera y Stanaiak (2013) agregan a éstos otros dos, el desarrollo de suvenires y el carácter inclusivo de la ruta. Repasemos los principales factores:

1. Coordinación de la ruta.

2. Apoyo del Gobierno.

3. Masa crítica de la oferta.

4. Diversidad de las experiencias y las historias ofrecidas.

5. Calidad de la oferta.

6. Consistencia con el tema de la ruta

7. Oferta y características de los suvenires.

8. Demanda del mercado.

9. Inclusión social.

\subsection{Coordinación de la ruta.}

La coordinación de la ruta es un factor clave para su éxito; es una tarea que da comienzo desde el inicio de su organización. La coordinación debe asumir responsabilidades fundamentales para la vida de la ruta. La carencia de una dirección precisa en la ruta ocasiona dificultades que conducen al fracaso de los proyectos.

Las dificultades nacen porque las rutas no son una propiedad individual sino colectiva que inicialmente cuentan con el apoyo de algunos emprendedores [sector privado] y el apoyo de operadores externos, [sector público]. Pero la 
experiencia indica que el esfuerzo principal en el territorio recae en una persona quien cumple un rol decisivo para el futuro de la ruta.

El coordinador debe resolver aspectos que suelen generar conflictos entre los miembros de la ruta tales como: quienes pueden integrarla, cumplimiento de las normas de calidad, relaciones con el gobierno e instituciones y mediar en los conflictos de intereses que se presenten.

Un inconveniente importante que puede aparecer para el destino de la ruta es que no siempre se compensa el trabajo de coordinador de la ruta para Ilevarla adelante, lo que no toma en cuenta que sin él, la ruta no tiene destino.

\subsection{Apoyos del Gobierno}

Las rutas alimentarias no existen independientemente de otros organismos de la comunidad, su organización refleja a la comunidad. La ruta y las instituciones de la comunidad conforman una red de interdependencia Mason (2010) y, por supuesto, las redes incluyen al gobierno local y a las autoridades de turismo.

Una ruta es un concepto dinámico en continua interacción entre sus miembros, incluyendo varios organismos que compiten y cooperan en los ámbitos local, estatal y nacional.

La ayuda del gobierno local es importante, en general las rutas se inician gracias al apoyo de los gobiernos. Sus contribuciones son variadas. Las agencias estatales y regionales de turismo pueden dar fuerte apoyo a productos que responden a sus propias prioridades, no lo hacen por benevolencia, sino porque tienen necesidad de contar con proyectos. (Mason, 2010)

El capital social de los impulsores de la ruta es fundamental para obtener los apoyos estales disponibles, sin un proyecto no es sencillo obtener los fondos públicos para desarrollar la ruta, por eso la capacidad de las universidades y del gobierno local para diseñarlo es clave. 


\subsection{Masa crítica de la oferta}

Constituye un factor clave de la ruta la masa crítica que la misma reúne. El destino turístico, o la ruta, no se crea por individuos aislados. El agrupamiento es una cuestión central y por lo tanto la cantidad agrupada y las características de los mismos son decisivas.

La masa crítica que convierte en viable a una ruta tiene dos aspectos a considerar, uno es la variedad de la oferta, en el sentido de contar con atractivos diversos, el otro contar con un número de empresas suficientemente grande como para que constituyan una oferta sólida.

Uno de los integrantes, propietario de un restaurante de una ruta al ser entrevistado sobre su conexión con la misma respondió: " .... La gente va a venir si piensa, que no va a viajar por una fantástica cena en un restaurante, sino que viaja por lo menos por tres fantásticas cenas en tres restaurantes" (Mason, 2010:165).

\section{La presentación conjunta de una serie de empresas independientes se} convierte en un destino en sí mismo, algo que los operadores individuales por sí mismos encontrarían difícil de lograr. La masa crítica es ir más allá de las empresas individuales para proporcionar una experiencia de destino que es mayor que la suma de las partes.

Contar con una masa crítica mínima también es importante porque facilita que los organismos oficiales tengan en consideración a la ruta y a sus integrantes. Finalmente los políticos no pueden hacer la vista ciega frente a un conglomerado importante pues restaría votos.

\subsection{Diversidad de las experiencias y las historias ofrecidas}

Los turistas no visitan una ruta del vino para tomar vino, sino para consumir una experiencia. Richards (2012) valora a la experiencia por sobre los servicios, afirma que "la economía de la experiencia" está por sobre la economía de los servicios". Interactuar con los turistas y brindarle protagonismo para que él construya su experiencia son una de las claves del éxito. 
En la búsqueda de la experiencia del turista subyace una demanda de educación y de conocimiento. La ruta debe contar una historia y hacerlo supone establecer una estrategia de diferenciación. Una historia es lo que hace a un establecimiento diferente de otro, esa historia propia, sin embargo, debe ser consistente con la historia general de la ruta.

¿Cómo construir la historia que un alimento debe contar? La trama argumental a contar debe alinearse con la que cuenta el territorio. De otra manera no sería consistente y difícilmente sería convincente. Duhart (2002) incluye entre los factores sobre los que se conforma la identidad de los alimentos, y que conforman una trama simbólica a los siguientes:

- El paisaje,

- La historia,

- Contextos culturales territoriales muy específicos [relación con expresiones artísticas, aspectos lúdicos, deportivos, religión, etc.] y

- El medio construido o el desarrollo tecnológico

Una forma, más pragmática, "más turística" de diseñar la historia, es la que proponen Mason y O'Mahony (2007) quienes lo hacen abordando las áreas que interesan al turista gastronómico:

- Salud

- Cocina

- Estilo de vida

- Regionalismo

- Medio ambiente y

- Ruralidad

La variedad de experiencias ofrecidas es muy importante. La gente no debe aburrirse visitando el mismo tipo de lugares, haciendo las mismas cosas y consumiendo el mismo tipo de comida. 


\subsection{Calidad de la oferta}

No basta con incorporarse a una ruta para tener éxito empresario. La empresa y la ruta tienen mejor fortuna cuando las empresas toman el compromiso con el turista como una cuestión importante. Respeto a los horarios de apertura, personal entrenado, contar una historia, y ofrecer experiencias a los visitantes resulta decisivo para la empresa y, a la vez, para la ruta.

La ruta está dirigida por las empresas tanto a consumidores como a turistas e implica responder con un alto nivel de calidad de los productos y servicios. Es mucho más que vender alimentos o vinos. A grandes rasgos, se requiere considerar los siguientes factores:

- Presentación. Adecuada para brindar la experiencia y respetuosa de las normas, etc.

- Servicios. Calidad de la comida y de la oferta en general, capacitación del personal, información, etc.

- Carreteras y carteles. Todos los aspectos que hacen al acceso al producto.

Para abordar esta temática el compromiso debe formalizarse volcando en los protocolos de calidad lo que los integrantes de la ruta públicamente se comprometen a cumplir.

Los protocolos de calidad de la ruta deben proteger sus claves de identidad, protección que debe considerar el carácter dinámico de la misma, dejando abierta por lo tanto la posibilidad de acomodar el protocolo en el largo plazo a las modificaciones que se produzcan.

\subsection{Consistencia con el tema de la ruta}

Numerosas rutas presentan itinerarios identificados por una producción emblemática sin que ésta participe como protagonista, a veces es sólo una referencia folletinesca que utiliza el prestigio del alimento a favor del turismo.

Con excesiva frecuencia las rutas carecen de un tema que les brinde coherencia más allá del vínculo de la denominación con la identidad del 
territorio. Se carece de una propuesta de actividades organizadas en torno al alimento o solamente se ofrece gastronomía y alguna visita al campo, se carece de una organización ad hoc y de un protocolo de calidad, no sólo se observa esta situación en América Latina, sino también en Francia (Frochot, 2000)

\subsection{Oferta y características de los suvenires}

Si la ruta alimentaria promueve el desarrollo de empresas y de nuevos usos para los productos de la ruta, como proponemos, desarrollar nuevos suvenires adquiere mucha importancia.

El suvenir, un producto tangible devenido en mercancía turística, que el comprador traslada fuera de la región, genera oportunidades para el desarrollo de emprendimientos novedosos. Sin el turista como cliente y sin las rutas alimentarias que le crean el mercado no existirían.

La característica del suvenir tiene importancia porque ésta delimita su mercado. Una remera grabada con el logo de la ruta difícilmente se comercialice fuera de su región. Un alimento elaborado, basado en el producto de la ruta, puede lograr un posicionamiento comercial fuera de su lugar de origen potenciando así a las empresas productoras.

El nivel de desarrollo de los suvenires de una ruta refleja el trabajo que se ha realizado promoviendo la creación de empresas y la innovación.

\subsection{Demanda}

Una ruta no debiera desarrollarse sin un claro enfoque de demanda. Uno de los problemas más comunes es el desarrollo de productos y destinos a los que luego no llegan turistas.

Evaluar el comportamiento de la ruta en el mercado constituye un punto de fundamental importancia para considerar el grado de éxito de la ruta. 


\subsection{Inclusión social}

La inclusión social es un aspecto de vital importancia para evaluar el comportamiento de las rutas alimentarias porque el desarrollo de los territorios rurales es siempre un objetivo indubitable de todo proyecto de turismo rural.

El turismo exitoso requiere centrarse en las personas y escuchar todas las voces del territorio. Una ruta, con la concepción que se ha presentado, supone ofrecer un explícito beneficio en favor de los pobladores más pobres, al menos no excluirlos. La inclusión social se verifica especialmente por el tamaño económico de los actores involucrados en la ruta.

Inclusión social no implica que la incorporación de los sectores más pequeños desaloje a las empresas grandes, por el contrario, cuando se logran sinergias entre los pequeños emprendedores y las grandes empresas la ruta será más rica en masa crítica y en diversidad.

Una de las fallas más notorias de algunos proyectos desarrollados en América Latina es que sólo integran en su organización y en la conducción política de la ruta a las empresas más grandes o a las de un único sector sin incorporar a los agricultores o dejándolos fuera de la conducción de la organización de la ruta. También es cierto que se observan intentos clasistas de construcción de rutas, con los que somos tan críticos como con los exclusivos.

\section{CONCLUSIONES}

Varios factores condicionan el éxito de mercado de una ruta alimentaria: que cuente con una coordinación efectiva y con apoyo del gobierno, que alcance una masa crítica de oferentes los que a su vez ofrezcan una diversidad de experiencias, que cuente con protocolos de calidad, que su oferta guarde consistencia con el tema que evoca, que logre desarrollar numerosos suvenires $y$, por supuesto, que encuentre su nicho de demanda en el mercado.

Desde nuestra posición, la de la ruralidad en América Latina, y con un enfoque que demanda que el turismo sea instrumento de transformación de la realidad, una ruta no será exitosa si no es inclusiva, pues éste es el factor de mayor relevancia para medir su éxito. 
Cuando se trabaja únicamente con sectores empresariales las coincidencias y las inversiones fluyen más fácilmente y los conflictos se atenúan. Naturalmente es más sencillo desarrollar proyectos turísticos asociando a empresas exitosas que hacerlo incluyendo campesinos o productores que frecuentemente no conocen del negocio turístico.

Las rutas alimentarias más reconocidas de América Latina, que han surgido con apoyos del Estado y de organismos internacionales, cuentan con productos turísticos novedosos, muchas veces sustentados en importantes inversiones, pero frecuentemente dejan a los agricultores fuera de la ruta y de su conducción. La Ruta de la Yerba Mate es una de las excepciones.

Los modelos exclusivos pueden mostrar éxito turístico, pero fracasan en mostrar al turismo como fuerza de transformación social. Con ellos no puede asegurarse si las rutas alimentarias son un instrumento para el desarrollo sostenible o, si por el contrario, lo son del statu quo.

Que el Estado disponga de recursos financieros para subsidiar a proyectos que sólo benefician a un conjunto reducido de empresas y deje fuera de las rutas a los productores que forjaron el paisaje cultural que da origen al negocio, cuestiona fuertemente su rol de mediador social.

La creencia en identidades con pretensiones totalizadoras, que niega la pluralidad de las identidades sociales es reduccionista y, en último término, puede ser un fundamento para la violencia (Sen, 2006). Reinterpretando a Sen, las configuraciones no inclusivas de las rutas alimentarias, que aprovechan los recursos territoriales para un solo sector social, un único eslabón de la cadena o un único componente del cluster llevan implícito un elevado riesgo, más aún en los países de América Latina signados por los conflictos agrarios.

Mucho tiempo demandó a la sociedad resolver un debate sobre la bondad o perversidad de un instrumento: el dinero. Hoy estamos seguros que el mismo es inocuo y que con su uso el hombre puede corromper o hacer el bien, puede tanto someter y esclavizar una sociedad como ofrecerle desarrollo e inclusión. El hombre elige. 
Jorge Luis Borges logró una bella síntesis vinculada al debate: "...nada hay menos material que el dinero, ya que cualquier moneda es, en rigor, un repertorio de futuros posibles."

Las rutas alimentarias son, a mi juicio, un instrumento inocuo. Según se diseñen e implementen cristalizarán la desigualdad o generarán desarrollo e inclusión social. Con seguridad ofrecen, también, un repertorio de esperanza para numerosas comunidades y culturas de la ruralidad latinoamericana.

\section{BIBLIOGRAFÍA}

Aguilar Criado E. (2005). Patrimonio y globalización: el recurso de la cultura en las Políticas de Desarrollo Europeas. Cuadernos de Antropología Social № 21.

Barrera E; Staniak C. (2013). Rutas Alimentarias. Una estrategia de desarrollo turístico inclusivo basada en alimentos con identidad. Turismo rural y conservación en la costa de Jalisco. En Turismo Rural y en áreas protegidas. Coordinadores J. de León Ledesma, M. M. González Hernández , C. J. León González y S. Moreno Gil. Editorial Síntesis. Madrid, España.

Barthes R. (1972). Mythologies, traducido por Annette Lavers. Nueva York: Hill and Wang. citado en Tobin (2002)

Bourdieu P. (1998). La distinción. Criterio y bases sociales del gusto. Madrid, Taurus.

Briedenhann J. y Wickens E. (2004): Tourism routes as a tool for the economic development of rural areas - vibrant hope or impossible dream?, Tourism Management, 25, 71-79.

Brillat-Savarin J. A. (2005). Fisiología del Gusto. Editorial Andrómeda.

Castedo L. (1999). Fundamentos culturales de la integración latinoamericana, Dolmen Ediciones, Caracas-Montevideo-Santiago de Chile. CEPAL (2007). Citado en Otone y Sojo (2008).

César Dachary A. y Arnaiz Burne S. M. (2006). Territorio y Turismo. Nuevas dimensiones y acciones. Universidad de Guadalajara. Centro Universitario de la Costa. México. 
De Certeau M., Giard L. y Mayol P. (2000). La invención de lo cotidiano. Habitar, cocinar. México D. F. pp. 176-177.

Desrochers P y Shimizu H. (2008). Yes, We Have No Bananas: A Critique of the "Food Miles" Perspective. Mercatus Policy Series Global Prosperity Initiative.

http://www.utsc.utoronto.ca/ facilities/documents/YesWeHaveNoBanana s_CritiqueoftheFoodMilePerspective.pdf - Acceso enero 2013.

Duhart F. (2002). Comedo Ergo Sum. Reflexiones sobre la identidad cultural alimentaria. Gazeta de Antropología, 18, artículo 15 • http://hdl.handle.net/10481/7403 - Acceso enero 2013

Elías L. V. (2006). El turismo del vino, otra experiencia de ocio. Bilbao. Editorial Deusto.

Frochot I. (2000) Food trails in France. WTO-CTO. Local food \& tourism international conference. Chipre. 9-11 November 2000.

Hall C. M. (2004). Small firms and wine and food tourism in New Zealand: issues of collaboration, clusters and lifestyles, pp.167-181 in Small Firms in Tourism: International Perspectives, (ed), R.Thomas, Elsevier, Oxford.

Hall C. M. y Mitchell R. (2000). Wine tourism in the Mediterranean: a tool for restructuring and development". Thunderbird International Business. Review, 424.

Hall M. (2012) Boosting food and tourism-related regional economic development. En En The OECD-Korea Workshop, OECD Studies on Tourism, OECD Publishing. http://dx.doi.org/10.1787/9789264171923-en pp $49-61$.

Haywood K. M. (1988): Responsible and responsive tourism planning in the community, Tourism Management, 9(2), 105-107.

Linck, T. 2006. La economía y la política de los territorios. ALASRU. Análisis Latinoamericano del medio rural. 3.

Long P. T., Perdue R. R. y Allen L. (1990). Rural resident perceptions and attitudes by community level of tourism, Journal of Travel Research, 19, 3-9. 
López-Guzmán Guzmán T y Sánchez Cañizares S. (2008). La creación de productos turísticos utilizando rutas enológicas. Pasos Vol. 6 № 2. Número Especial Turismo Gastronómico y Enoturismo.

MacCannell D. (2003). El Turismo. Una nueva teoría de la clase ociosa. Editorial Melusina. España.

Mason R. y O'Mahony B. (2007). On the trail of food and wine: the tourist search for meaningful experience, Annals of Leisure Research, vol. 10, no. 3/4, pp. 498-517. Citado en Mason 2010.

Mason Robert J. (2010). Thesis submitted in fulfilment of the requirements of the Doctorate of Philosophy. Victoria University School of International Business Melbourne, Australia. http://vuir.vu.edu.au/16039/1/robert_mason_phd.pdf - Acceso enero 2013.

Mc Crone D., Morris A. y Keily R. (1995). Scotland the Brand: The Making of Scottish Heritage Edinburgh: EUP. Citado en Burnett, Kathryn Anne 2000. Local food \& tourism international conference. Larnaka, Chipre. 911 November 2000.

Mendelson R. y Quick D. The Napa Valley: an emblematic California Coastal Valley vineyard landscape (USA) http://www.icomos.org/studies/viticoles/viticole12.pdf - Acceso agosto 2012.

Muchnik J. (2010). Alimentos y poesía. Presentación realizada para el $250 \cong$ aniversario de la Academia de Agricultura de Francia. Sesión "Cultura y Gastronomía" Traducción/adaptación de la versión francesa realizada por el autor.

Pasos, Volumen 6, No. 2 de abril de 2008. www.pasosonline.org

Prentice R. (1993). Community-driven tourism planning and residents' preferences, Tourism Management, 14, 218-227.

Renting H., Oostindie H., Laurent C., Brunori G., Barjolle D., Moxnes Jervell A., Granberg L. y Heinonen M. (2003). Multifunctionality of agricultural activities, changing rural identities and new institutional arrangements Int. J. Agricultural Resources, Governance and Ecology, Vol. 7, No. 4. 
Richards G. (2012). An overview of food and tourism trends and policies. Chapter 1. En The OECD-Korea Workshop, OECD Studies on Tourism, OECD Publishing. http://dx.doi.org/10.1787/9789264171923-en pp 1443.

Schlüter R. G. (2002). The immigrant's heritage in South America: food and culture as a new sustainable tourism product, Faculty of Arts and Science, Harvard University, http://www.fas.harvard.edu/ drclas/publications/revista/Tourism/Schluter. html - Acceso febrero 2013.

Sen Amartya (2000). Desarrollo y libertad. Editorial Planeta. España.

Tobin J. (2002). Patrimonializaciones gastronómicas. La Construcción Culinaria de la Nacionalidad. En La cocina como patrimonio (in) tangible. Primeras Jornadas de Patrimonio Gastronómico. Comisión para la Preservación del Patrimonio Histórico Cultural de la Ciudad de Buenos Aires.

WTO-CTO (2000). Local food \& tourism international conference. Chipre. 9-11 November 2000. 